\title{
COMPORTAMENTO DA PITANGUEIRA (Eugenia uniflora L) SOB IRRIGAÇÃO NA REGIÃo dO VALE DO RIO MOXOTÓ, PERNAMBUCO ${ }^{1}$
}

\author{
JOÃO EMMANOEL FERNANDES BEZERRA², ILDO ELIEZER LEDERMAN³, JOSUÉ FRANCISCO DA SILVA JÚNIOR, \\ MARTA ASSUNÇÃO ALVES 5
}

\begin{abstract}
RESUMO - O cultivo da pitangueira no Nordeste vem crescendo rapidamente devido à utilização do seu fruto pelas indústrias de polpas e sucos; no entanto, a ausência de cultivares adaptadas às condições irrigadas constitui atualmente um dos principais problemas da cultura. O presente trabalho foi conduzido com o objetivo de estudar o comportamento de dez dentre os 85 acessos da Coleção de Germoplasma de Pitangueira do IPA, sob irrigação, em Ibimirim, na região semi-árida de Pernambuco. Foram avaliadas características de crescimento, rendimento e qualidade do fruto de plantas oriundas de "pé-franco" e enxertadas. A pitangueira conduzida sob irrigação mostrou boa adaptação às condições do Vale do Rio Moxotó, tanto para as características de crescimento como de produção e qualidade do fruto. Os acessos mostraram grande variabilidade em relação às características físicas e químicas do fruto. Foram identificados dois acessos promissores ${ }^{-}$IPA-13.2 e IPA-1.1E ${ }^{-}$, os quais reuniram as melhores características de produção e de qualidade do fruto.
\end{abstract}

Termos para indexação: Pitanga, seleção, irrigação, adaptação regional, cultivar.

\section{PERFORMANCE OF SURINAM CHERRY (Eugenia uniflora L.) UNDER IRRIGATION IN THE MOXOTÓ VALLEY, PERNAMBUCO STATE, BRAZIL}

\begin{abstract}
Surinam cherry crop is growing fastly in the Northeast region of Brazil due to its fruit uses by the industry in processing juice and pulp. However, one of the main problems of this crop is the lack of an apropriated cultivar adapted to such climate environments and irrigated conditions. This work was carried out aiming to study the performance of ten among 85 Surinam cherry accesses of IPA's germplasm collection cultivated under drip irrigation at the semi-arid region of Pernambuco State. The following characteristics were evaluated: growth, yield and fruit quality of plants originated from seedlings as well as from grafting. Surinam cherry trees have shown good adaptability to such environmental conditions, both in respect to plant growth characteristics as well as to yield and fruit quality. Accesses have shown great variability in relation to physical-chemical characteristics of fruits. Two promising materials were identified ${ }^{-}$IPA-13.2 and IPA-1.1E, with better yielding and fruit quality characteristics.
\end{abstract}

Index terms: Pitanga, selection, irrigation, plant adaptation, cultivar.

A pitangueira (Eugenia uniflora L.), frutífera nativa da região que se estende desde o Brasil Central até o norte da Argentina, tem sido largamente disseminada por outras regiões tropicais e subtropicais do mundo (Fouqué, 1981; Williams et al., 1987). O seu cultivo no Estado de Pernambuco vem crescendo em razão da utilização do fruto para o preparo de polpa e suco, como também para a fabricação de sorvetes, refrescos, geléias, licores e vinhos (Lederman et al., 1992; Bezerra et al., 2000).

Embora a pitangueira apresente grande potencial econômico de exploração para a fruticultura do Nordeste, as pesquisas dirigidas à cultura, principalmente nas áreas irrigadas, são muito escassas.

Assim, a maioria dos pomares existentes não utiliza cultivares definidas e são geralmente provenientes de plantas propagadas por sementes, resultando em grande variabilidade, originada pelo processo de recombinação gênica decorrente da utilização desse tipo de muda na formação dos pomares.

Por outro lado, para se obter cultivares com características definidas, são necessários estudos de caracterização e de avaliação de germoplasma, com a finalidade de se identificarem genótipos com características desejáveis. Esses genótipos poderão ser indicados para plantios comerciais e também utilizados em hibridações, a fim de gerar populações segregantes, onde se fará a seleção de materiais que reúnam as características desejadas (Lopes et al., 2000).

A importância de introduzir, caracterizar e avaliar germoplasma de fruteiras em ambientes diversificados, como etapas no processo de melhoramento genético, tem sido evidenciada também por diversos autores (Dudley \& Moll, 1969; Vilela-Morales, 1990; Gonzaga Neto, 1995).

Bezerra et al. (1995, 1997a), avaliando 122 acessos de pitangueira na Zona da Mata Norte de Pernambuco, em condições de sequeiro, relacionaram dez genótipos com elevado potencial produtivo e boas características agronômicas. Após avaliação clonal, o acesso IPA-2.2 foi lançado como a primeira cultivar comercial brasileira, sob a denominação de 'Tropicana', a qual apresenta como principais vantagens a alta produção- $20,8 \mathrm{~kg}$ de frutos/ano (média de dez anos), peso médio do fruto variando de 3 a 4,5g, polpa avermelhada e relação SST/Acidez de 4,1 (IPA, 2000).

Este trabalho foi realizado com o objetivo de caracterizar, avaliar e selecionar genótipos da coleção de pitangueira do IPA, no Vale do Moxotó, sob condições irrigadas, visando à recomendação de cultivares comerciais.

O trabalho foi realizado na Área Experimental da Empresa Pernambucana de Pequisa Agropecuária - IPA, em Ibimirim, situada no Vale do Rio Moxotó, na região semi-árida de Pernambuco. As coordenadas geográficas são $8^{\circ} 32$ ' $15^{\prime \prime}$ de latitude Sul e 3741'30" de longitude Oeste e uma altitude $431 \mathrm{~m}$. O clima é do tipo B'Swh', semiárido muito quente tipo estepe (classificação de Köeppen), com precipitação pluvial e temperaturas médias anuais de $420 \mathrm{~mm} \mathrm{e} 25^{\circ} \mathrm{C}$, respectivamente. $\mathrm{O}$ solo da área é classificado como Solonetz solodizado.

A coleção é constituída de 85 acessos, duplicados do Banco de Germoplasma de Pitangueira do IPA, na Estação Experimental de Itambé, sendo 74 originados de mudas do tipo "pé-franco" e implantados em julho de 1992 e 11 de mudas enxertadas introduzidas em agosto de 1994. Cada acesso é representado por uma planta, e apenas as dez melhores seleções estão apresentadas neste trabalho.

O manejo agronômico do pomar obedeceu às recomendações gerais citadas por Bezerra et al. (1997b, 2000), e o sistema de irrigação utilizado foi o de xique-xique (Sistema de irrigação localizada, que consiste na aplicação de água, através de tubos perfurados, com diâmetro de furo de, no máximo, 1,6mm), com intervalos entre regas de dois a três dias, baseado em dados meteorológicos e visando a atender as exigências hídricas da cultura.

Os dados de produção correspondem às colheitas realizadas no período de 1999 a 2001. As avaliações foram realizadas em cada matriz,

\footnotetext{
${ }^{1}$ (Trabalho 141/2003). Recebido: 03/10/2003. Aceito para publicação: 23/03/2004.

${ }^{2}$ Eng. Agr., M.Sc., bolsista CNPq, Empresa Pernambucana de Pesquisa Agropecuária - IPA, Caixa Postal 1022, CEP 5076-000, Recife, PE. E-mail: emmanoel@ ipa.br; ${ }^{3}$ Eng. Agr., PhD., Empresa Brasileira de Pesquisa Agropecuária - Embrapa/IPA, bolsista CNPq. E-mail: ildo@ipa.br;

${ }^{4}$ Eng. Agr., M.Sc., IPA, bolsista da Fundação de Amparo à Ciência e Tecnologia de Pernambuco - FACEPE. E-mail: josuéf@ipa.br

${ }^{5}$ Eng.Agr., M.Sc., IPA; E-mail: martaaa@ipa.br
} 
tomando por base a amostra de uma planta para as análises de crescimento (altura da planta e diâmetros do caule e da copa), fenológica (época de colheita) e de produção. Para a avaliação das características físico-químicas do fruto (pesos do fruto, das sementes e da polpa; diâmetros transversal e longitudinal; teor de sólidos solúveis totais SST; e acidez total titulável - ATT), foram utilizados 20 frutos de cada acesso, conforme procedimentos descritos por Bezerra et al. (1997a).

Os resultados das avaliações que se seguem correspondem a pitangueiras com nove anos (plantas provenientes de pé-franco) e sete anos (plantas enxertadas) de idade. A altura das plantas originadas de sementes variou de 2,2m (IPA-14.3) a 2,9m (IPA-13.2). A altura das matrizes enxertadas não apresentou variação, uma vez que todas as seleções externaram altura média de 2,0m (Tabela 1). Esse comportamento devese, possivelmente, à diferença de idade entre as plantas oriundas de pés-francos e as enxertadas.

TABELA 1 - Características de crescimento de dez seleções de pitangueiras oriundas de pé-franco e enxertadas com nove e sete anos de idade, respectivamente, em Ibimirim - PE, 2001.

\begin{tabular}{cccc}
\hline Seleção & $\begin{array}{c}\text { Altura da } \\
\text { Planta (m) }\end{array}$ & $\begin{array}{c}\text { Diâmetro do } \\
\text { Caule (cm) }\end{array}$ & $\begin{array}{c}\text { Diâmetro da } \\
\text { Copa (m) }\end{array}$ \\
\hline Plantas Pés-Francos & & & \\
IPA 3.1 & 2,6 & 8,0 & 4,0 \\
IPA 7.1 & 2,5 & 9,1 & 3,8 \\
IPA 13.2 & 2,9 & 10,5 & 4,0 \\
IPA 14.3 & 2,2 & 8,5 & 3,4 \\
IPA 15.3 & 2,7 & 9,5 & 3,2 \\
\hline Média & 2,6 & 9,1 & 3,7 \\
\hline Plantas Enxertadas & & & \\
IPA 1.1 E & 2,0 & 10,2 & 3,0 \\
IPA 2.2 E & 2,0 & 8,8 & 3,3 \\
IPA 3.1 E & 2,0 & 8,6 & 3,2 \\
IPA 7.3 E & 2,0 & 8,1 & 3,3 \\
IPA 11.3 E & 2,0 & 7,5 & 3,3 \\
\hline Média & 2,0 & 9,0 & 3,2 \\
\hline
\end{tabular}

O diâmetro do caule, de forma geral, variou de $8,0 \mathrm{~cm}$ (IPA-3.1) a $10,5 \mathrm{~cm}$ (IPA-13.2) nas plantas de pé-franco, e de 7,5cm (IPA-11.3E) a 10,2cm (IPA-1.1E) nas enxertadas. O maior diâmetro da copa das plantas de pé-franco foi registrado nas seleções IPA-3.1 e IPA-13.2 (ambas com $4,0 \mathrm{~m})$ e o menor, na IPA-15.3 (3,2m). Com relação às plantas enxertadas, a variação foi de 3,0m (IPA-1.1E) a 3,3m (IPA-2.2E, IPA-7.3E e IPA-11.3E). Resultados semelhantes foram obtidos por Bezerra et al. (1997a) em plantas com a mesma idade cultivadas na Zona da Mata de Pernambuco.

Observou-se que, de maneira geral, as produções médias das plantas originadas de pé-franco $(33,1 \mathrm{~kg}$ de frutos/ano) foram $26,1 \%$ mais altas do que as enxertadas (24,5 kg de frutos/ano), (Tabela 2). A diferença da idade entre as plantas propagadas de formas distintas explica a maior produção das seleções de pé-franco. As dez matrizes
TABELA 2 - Produção de dez seleções de pitangueiras oriundas de péfranco e enxertadas no período de 1999 a 2001, IbimirimPE.

\begin{tabular}{|c|c|c|c|c|c|}
\hline \multirow[b]{2}{*}{ Seleção } & \multicolumn{3}{|c|}{ Produção (kg) } & \multirow[b]{2}{*}{ Total } & \multirow[b]{2}{*}{ Média } \\
\hline & 1999 & 2000 & 2001 & & \\
\hline \multicolumn{6}{|c|}{ Plantas Pés-Francos } \\
\hline IPA 3.1 & 42,8 & 27,2 & 27,3 & 97,3 & 32,4 \\
\hline IPA 7.1 & 37,9 & 38,5 & 21,4 & 97,8 & 32,6 \\
\hline IPA 13.2 & 40,6 & 55,1 & 24,7 & 120,4 & 40,1 \\
\hline IPA 14.3 & 31,7 & 39,5 & 21,9 & 93,1 & 31,0 \\
\hline IPA 15.3 & 34,1 & 40,5 & 13,2 & 87,8 & 29,3 \\
\hline Média & 37,4 & 40,2 & 21,7 & 99,3 & 33,1 \\
\hline \multicolumn{6}{|c|}{ Plantas Enxertadas } \\
\hline IPA $1.1 \mathrm{E}$ & 42,3 & 27,0 & 16,0 & 85,3 & 28,4 \\
\hline IPA $2.2 \mathrm{E}$ & 34,6 & 23,3 & 14,1 & 72,0 & 24,0 \\
\hline IPA $3.1 \mathrm{E}$ & 29,7 & 22,8 & 11,5 & 64,0 & 21,3 \\
\hline IPA $7.3 \mathrm{E}$ & 25,6 & 31,4 & 21,6 & 78,6 & 26,2 \\
\hline IPA $11.3 \mathrm{E}$ & 13,7 & 36,8 & 16,8 & 67,3 & 22,4 \\
\hline Média & 29,2 & 28,3 & 16,0 & 73,4 & 24,5 \\
\hline
\end{tabular}

apresentaram variabilidade na produção, com valores entre 29,3 e 40,1 $\mathrm{kg}$ de frutos/ano para as seleções de pé-franco, e entre 21,3 e 28,4 kg de frutos/ano nas enxertadas. Esses resultados obtidos foram superiores àqueles encontrados por Bezerra et al. (1997a), em trabalho conduzido sob regime de sequeiro, na Zona da Mata Norte de Pernambuco, onde ocorre um período de déficit hídrico que se estende de setembro a fevereiro, e as condições ambientais são bastante distintas. Além disso, a interação entre o genótipo e o meio ambiente também deve ser considerada.

De acordo com a Tabela 3, pode observar-se que existe um período de oito meses de produção contínua, estendendo-se de maio a dezembro, e que corresponde a $98,5 \%$ do total de frutos produzidos no ano e com pico no mês de novembro. Nos demais meses, as produções foram insignificantes. Esse comportamento das plantas sob irrigação diferiu daquele sob condições de sequeiro, em que as plantas apresentaram dois períodos de colheita, um de abril a maio $(21,9 \%)$ e outro mais significativo, indo de agosto a outubro, com 60,8\% do total produzido (Bezerra et al., 1995, 1997a).

As seleções mostraram variabilidade quanto ao peso e composição do fruto, teor de SST e ATT (Tabela 4). Os pesos médios do fruto variaram de 3,3g (IPA-1.1E) a 6,9g (IPA-11.3E) e, em geral, foram superiores aos de pitangueiras avaliadas em Itambé, na região da Mata

TABELA 3 - Distribuição média porcentual da produção de dez seleções de pitangueiras no período de 1999 a 2001, Ibimirim - PE.

\begin{tabular}{|c|c|c|c|c|c|c|c|c|c|c|c|c|}
\hline \multirow[b]{2}{*}{ Seleção } & \multicolumn{12}{|c|}{ Meses } \\
\hline & Jan. & Fev. & Mar. & Abr. & Maio & Jun. & Jul. & Ago. & Set. & Out. & Nov. & Dez. \\
\hline \multicolumn{13}{|c|}{ Plantas Pés-Francos } \\
\hline IPA 3.1 & 0,4 & 0,0 & 0,0 & 0,2 & 7,8 & 7,2 & 13,3 & 17,3 & 9,8 & 24,5 & 10,2 & 9,5 \\
\hline IPA 7.1 & 0,5 & 0,3 & 0,0 & 0,9 & 12,0 & 10,2 & 9,7 & 18,2 & 6,3 & 12,4 & 20,1 & 9,4 \\
\hline IPA 13.2 & 1,7 & 0,1 & 0,0 & 1,1 & 5,0 & 15,4 & 14,1 & 14,7 & 5,7 & 8,6 & 26,2 & 7,2 \\
\hline IPA 14.3 & 0,5 & 0,0 & 0,0 & 0,2 & 13,2 & 7,5 & 6,1 & 15,5 & 11,7 & 7,5 & 17,0 & 20,7 \\
\hline IPA 15.3 & 4,2 & 0,0 & 0,0 & 0,0 & 0,3 & 9,9 & 1,9 & 12,7 & 8,5 & 7,6 & 43,5 & 11,5 \\
\hline \multicolumn{13}{|c|}{ Plantas Enxertadas } \\
\hline IPA $1.1 \mathrm{E}$ & 0,5 & 0,0 & 0,0 & 0,3 & 9,2 & 13,1 & 10,0 & 17,3 & 9,6 & 10,0 & 17,4 & 12,5 \\
\hline IPA $2.2 \mathrm{E}$ & 0,6 & 0,0 & 0,0 & 0,1 & 9,3 & 13,6 & 15,9 & 6,4 & 3,4 & 24,0 & 18,3 & 8,4 \\
\hline IPA $3.1 \mathrm{E}$ & 0,0 & 0,0 & 0,0 & 2,6 & 2,5 & 7,6 & 5,5 & 3,2 & 5,9 & 21,7 & 31,9 & 19,1 \\
\hline IPA $7.3 \mathrm{E}$ & 0,8 & 0,1 & 0,0 & 0,1 & 3,5 & 9,4 & 4,6 & 12,9 & 17,2 & 24,8 & 18,1 & 8,6 \\
\hline IPA $11.3 \mathrm{E}$ & 0,0 & 0,0 & 0,0 & 0,0 & 3,6 & 11,2 & 17,4 & 14,1 & 5,4 & 13,3 & 24,4 & 10,6 \\
\hline Média & 0,9 & 0,1 & 0,0 & 0,6 & 6,6 & 10,5 & 9.9 & 13,2 & 8,4 & 15,4 & 22,7 & 11,8 \\
\hline
\end{tabular}


TABELA 4 - Características físicas e químicas do fruto de dez seleções de pitangueiras, Ibimirim - PE, 2001.

\begin{tabular}{|c|c|c|c|c|c|c|c|}
\hline \multirow[b]{2}{*}{ Seleção } & \multirow{2}{*}{$\begin{array}{l}\text { Peso do fruto } \\
\text { (g) }\end{array}$} & \multicolumn{2}{|c|}{ Composição do fruto (\%) } & \multirow[b]{2}{*}{ pH } & \multirow[b]{2}{*}{ SST ( ${ }^{\circ}$ Brix) } & \multirow[b]{2}{*}{ ATT (\%) } & \multirow[b]{2}{*}{ SST/ATT } \\
\hline & & Polpa & Sementes & & & & \\
\hline \multicolumn{8}{|c|}{ Plantas Pés-Francos } \\
\hline IPA 3.1 & 5,7 & 83,2 & 16,8 & 2,9 & 12,2 & 1,6 & 7,6 \\
\hline IPA 7.1 & 3,6 & 76,9 & 23,1 & 3,0 & 9,6 & 1,7 & 5,6 \\
\hline IPA 13.2 & 4,5 & 83,1 & 16,9 & 3,1 & 9,0 & 1,5 & 6,0 \\
\hline IPA 14.3 & 4,0 & 74,2 & 25,8 & 2,9 & 9,0 & 1,4 & 6,4 \\
\hline IPA 15.3 & 4,9 & 78,7 & 21,3 & 3,0 & 13,4 & 1,7 & 7,9 \\
\hline \multicolumn{8}{|c|}{ Plantas Enxertadas } \\
\hline IPA $1.1 \mathrm{E}$ & 3,3 & 81,0 & 19,0 & 3,1 & 14,4 & 1,6 & 9,0 \\
\hline IPA $2.2 \mathrm{E}$ & 3,8 & 74,7 & 25,3 & 2,9 & 10,0 & 1,5 & 6,7 \\
\hline IPA $3.1 \mathrm{E}$ & 4,3 & 64,6 & 35,4 & 3,0 & 11,2 & 1,4 & 8,0 \\
\hline IPA $7.3 \mathrm{E}$ & 4,5 & 81,1 & 18,9 & 2,9 & 10,6 & 1,8 & 5,9 \\
\hline IPA $11.3 \mathrm{E}$ & 6,9 & 82,0 & 18,0 & 3,1 & 13,4 & 1,4 & 9,6 \\
\hline Média & 4,5 & 77,9 & 22,1 & 3,0 & 11,3 & 1,6 & 7,3 \\
\hline
\end{tabular}

Norte de Pernambuco (Bezerra et al., 1997a). O rendimento de polpa esteve acima de $80 \%$ em cinco dos dez acessos, com destaque para a seleção IPA-3.1 (83,2\%), que também foi uma das mais produtivas. Não houve grandes variações no $\mathrm{pH}$, cujos valores registrados estiveram entre 2,9 e 3,1. Quanto ao teor de SST, os acessos apresentaram médias entre $9,0^{\circ}$ Brix (IPA-13.2 e IPA-14.3) e 14,4 ${ }^{\circ}$ Brix (IPA-1.1E). Além deste, os acessos IPA-15.3 e IPA-11.3E também produziram frutos muito doces $\left(13,4^{\circ}\right.$ Brix). No que concerne à ATT, houve variação de 1,4 (IPA-14.3, IPA-3.1E e IPA11.3E) a 1,8\% (IPA-7.3E), e quanto à relação SST/ATT, que é um dos mais importantes parâmetros de qualidade, todos os acessos apresentaram valores superiores $(5,6$ a 9,6) ao da cultivar Tropicana testada na Zona da Mata $(4,1)$, conforme observações de Bezerra et al. (1997a).

Os acessos mostraram variabilidade em relação às características físicas e químicas do fruto.

As seleções IPA-13.2, IPA-1.1E e IPA-11.3E reuniram as melhores características de produção e de qualidade do fruto.

\section{REFERÊNCIASBIBLIOGRÁFICAS}

BEZERRA, J.E.F.; LEDERMAN, I.E.; PEDROSA, A.C.; DANTAS, A.P.; FREITAS, E.V. de. Performance of Surinam cherry (Eugenia uniflora L.) in Pernambuco, Brazil. Acta Horticulturae, Wageningen, n. 370, p. 77-81, 1995.

BEZERRA, J.E.F.; FREITAS, E.V. de.; LEDERMAN, I.E.; DANTAS, A.P. Performance of Surinam cherry (Eugenia uniflora L.) in Pernambuco, Brazil II - Productive period 1989-1995. Acta Horticulturae, Leuven, n. 452, p. 137-142, 1997a.

BEZERRA, J.E.F.; LEDERMAN, I.E.; PEDROSA, A.C.; MOURA, R.J.M. de; DANTAS, A.P. Recomendações para o cultivo de fruteiras tropicais. Recife, PE: IPA, 1997b, 64p. (IPA. Documentos, 24).

BEZERRA, J.E.F.; SILVA JÚNIOR, J.F. da; LEDERMAN, I.E. Pitanga (Eugenia uniflora L.). Jaboticabal: FUNEP, 2000. 30p. (Série Frutas Nativas, 1).

DUDLEY, J.W.; MOLL, R.H. Interpretation and use of estimates of heritability and genetic variances in plant breeding. Crop Science, Madison, n. 9, p. 257-262, 1969.

FOUQUÉ, A. Les plantes médicinales présentes en Fôret Guyanaise. Fruits, Paris, v.36, n.10, p. 567-592, 1981.

GONZAGA NETO, L. Melhoramento genético da aceroleira. In: SÃO JOSÉ, A.R.; ALVES, R.E. (eds.). Acerola no Brasil: produção e mercado. Vitória da Conquista-BA: UESB-DFZ, 1995, p. 15-21.

IPA(Empresa Pernambucana de Pesquisa Agropecuária). Pitanga cultivar tropicana. Recife, 2000. 4p. (Fôlder).

LEDERMAN, I.E.; BEZERRA, J.E.F.; CALADO, G. A pitangueira em Pernambuco. Recife: IPA, 1992. 20p. (Documentos, 19).

LOPES, R.; BRUCKNER, C.H.; FINGER, F.L.; LOPES, M.T.G. Avaliação de características do fruto de acessos de aceroleira. Revista Ceres, Viçosa, v. 47, n. 274, p. 627-638, 2000.

VILELA-MORALES, E.A. Documentação de recursos genéticos em fruticultura. In: SIMPÓSIO LATINO-AMERICANO SOBRE RECURSOS GENÉTICOS DE ESPÉCIES HORTÍCOLAS, 1., 1989, Campinas, SP. Anais... Campinas: Fundação Cargill, 1990. p. 128139.

WILLIAMS, C.N.; CHEW, W.Y.; RAJARATNAM, J.A. Tree and field crops of the wetter regions of the tropics. Harlow, England: Longman Scientific \& Technical, 1987.262p. 